\title{
A model for grand minima and geomagnetic reversals
}

\author{
D. D. Sokoloff ${ }^{1}$, G. S. Sobko ${ }^{2}$, V. I. Trukhin ${ }^{3}$ and V. N. Zadkov ${ }^{4}$ \\ Department of Physics, Moscow State University, 119991, Moscow, Russia \\ email: ${ }^{1} \mathrm{~d}_{-}$sokoloff@hotmail.com \\ ${ }^{2}$ sobko@physics.msu.ru \\ ${ }^{3}$ dean@phys.msu.ru \\ ${ }^{4}$ zadkov@phys.msu.ru
}

\begin{abstract}
We suggest a simple dynamical system which mimics a nonlinear dynamo which is able to provide (in specific domains of its parametric space) the temporal evolution of solar magnetic activity cycles as well as evolution of geomagnetic field including its polarity reversals. A qualitative explanation for the physical nature of both phenomena is presented and discussed.
\end{abstract}

Keywords. Sun: activity, Earth, magnetic fields, sunspots.

\section{Introduction}

Temporal behaviour of solar magnetic activity is far from just a cycle. Discussions of various features of long-term dynamics of solar activity including Maunder minimum and other Grand minima are presented in many papers included in this volume. Temporal behaviour of geomagnetic field is obviously specific. In contrast to the solar magnetic cycle, geomagnetic dipole field remains usually more or less constant and its secular and more long-term archaeomagnetic variations do not result in its polarity reversals. In geological timescales however geomagnetic field suddenly changed, as geologists believe, its polarity many times (Christensen et al. 2010; Hulot et al., 2010). Sequence of geomagnetic reversals as well as that one of solar Grand minima look aperiodic and irregular.

Various solar dynamo models including those discussed in this volume reproduce at least qualitatively solar Grand minima as well as geodynamo model based on direct numerical simulations give hundreds of reversals (Olson et al. 2010). Magnetic field reversals similar to some extent to the geomagnetic reversals was reproduced in laboratory dynamo experiments (Berhanu et al. 2007). Physical nature of these phenomena remains however not completely clear, cf. Choudhuri (2012).

Solar magnetic activity as well as geomagnetic field are thought to be driven by dynamos in spherical shells based on differential rotation and mirror-asymmetric convection. Particular manifestations of these dynamos are obviously specific. However it looks attractive to present both associated long-term dynamics as manifestations of a unique physical mechanism acting in two separate domains of the parametric space of dynamo governing parameters. Here we present a simple model which demonstrates a mechanism which can be responsible for both long-term dynamics.

We consider fluctuations in regeneration rate of poloidal magnetic field from toroidal one as the physical driver underlying mechanism leading to the magnetic long-term dynamics. To be specific, we discuss this mechanism in the framework of dynamo based on the classical $\alpha$-effect. However the idea looks applicable as well to the dynamos based on meridional circulation. 
$\alpha$-coefficient being a result of averaging over an ensemble of a moderate number of convective cells (say, $N \approx 10^{4}$ ) contains a noisy component which importance for dynamo was stressed e.g. by Hoyng (1993). Hoyng (1993) supposed correlation time and length of $\alpha$-fluctuations to be comparable with that ones for convective vortexes. Then one need fluctuations amplitude comparable with mean value of $\alpha$ to get an interesting long-term dynamics for dynamo generated magnetic field. Basing on the later results of direct numerical simulations of $\alpha$-effect, (Brandenburg \& Sokoloff 2002; OtmianowskaMazur, Kowal \& Hanasz 2006), determinations of $\alpha$-coefficient from dynamo shell models (Frick, Stepanov \& Sokoloff 2006) as well as laboratory measurements of the $\alpha$-coefficient (Stepanov et al., 2006) we presume that the correlation time and length of $\alpha$-fluctuations are comparable for the cycle period and size of solar convective zone; standard $N^{-1 / 2}$ estimation for the fluctuation size do not contradict to this presumption. Then we need $\delta \alpha / \alpha \approx 10 \%-20 \%$ to get a desired behaviour for dynamo generated field (Moss et al. 2008; Usoskin, Sokoloff \& Moss 2009).

\section{Dynamical system}

We obtain the desired simple model from general Parker mean-field dynamo models with differential rotation and $\alpha$-effect decomposing it in the Fourier series and truncating the series keeping in consideration as small number of modes as possible to get generation of magnetic field with nonvanishing magnetic moment (see Nefedov \& Sokoloff 2010, for details of calculations). More explicitly, we represent toroidal magnetic field $B$ and toroidal component of magnetic potential $A$ as

$$
B=-b_{1} \sin 2 \theta+b_{2} \sin 4 \theta, \quad A=a_{1} \cos \theta-a_{2} \cos 3 \theta,
$$

where $\theta$ is co-latitude and truncate Parker (1955) dynamo equation accordingly to get the following dynamical system

$$
\begin{gathered}
\frac{d a_{1}}{d t}=\frac{R_{\alpha} b_{1}}{2}-a_{1}-\frac{3 R_{\alpha} b_{1}}{8}\left(b_{1}^{2}+2 b_{2}^{2}\right), \\
\frac{d a_{2}}{d t}=\frac{R_{\alpha}}{2}\left(b_{1}+b_{2}\right)-9 a_{2}-\frac{3 R_{\alpha}\left(b_{1}+b_{2}\right)}{8}\left(b_{1}^{2}+b_{1} b_{2}+b_{2}^{2}\right),
\end{gathered}
$$

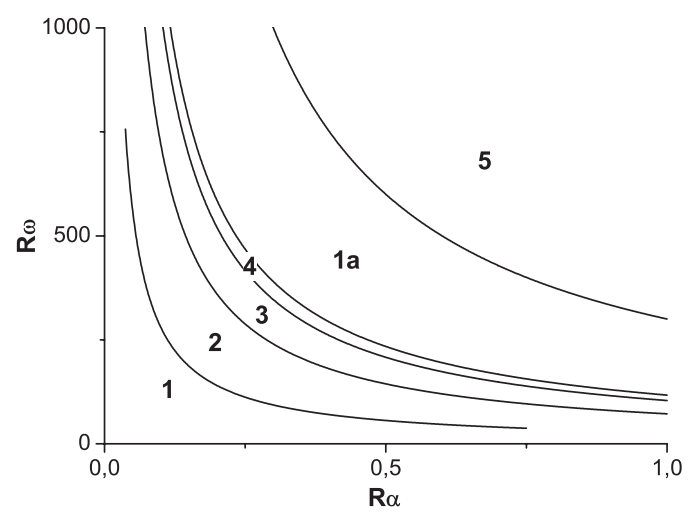

Figure 1. Synoptic map for dynamo regimes: 1, 1a - decay, 2 - stationary field, 3 vacillations, 4 - dynamo bursts, 5 - oscillations. 


$$
\begin{gathered}
\frac{d b_{1}}{d t}=\frac{R_{\omega}}{2}\left(a_{1}-3 a_{2}\right)-4 b_{1}, \\
\frac{d b_{2}}{d t}=\frac{3 R_{\omega} a_{2}}{2}-16 b_{2} .
\end{gathered}
$$

Here $R_{\alpha}$ and $R_{\omega}$ are dimensionless numbers for intensity of $\alpha$-effect and differential rotation correspondingly. We presume that the radial rotation shear is latitude independent and dominate over the latitudinal one and $\alpha(\theta) \sim \sin \theta$. We presume simple algebraic $\alpha$-quenching in form $\alpha \sim 1 /\left(1+B^{2} / B_{\text {eq }}^{2}\right)$ and measure magnetic field in units of the equipartition magnetic field, so $B_{\mathrm{eq}}=1$. We neglected regeneration of toroidal field from poloidal one due to the $\alpha$-effect and connect this regeneration with differential rotation only, i.e. consider so-called $\alpha \omega$-dynamo. Because the dynamo generated toroidal magnetic field is usually much larger then the poloidal one, we neglect in dynamical system nonlinear terms with $a_{1}$ and $a_{2}$. Replacing variables one can combine dimensionless numbers $R_{\alpha}$ and $R_{\omega}$ in so-called dynamo number $D=R_{\alpha} R_{\omega}$. Note that the coefficient $a_{1}$ is proportional to the magnetic moment of dynamo generated magnetic field.

\section{Dynamo regimes}

We simulate the dynamical system (2.2-2.5) numerically to isolate the following regimes of magnetic field evolution.

Let us start with dynamo regimes which occur for a time-independent $\alpha$-effect parameterized by dimensionless number $R_{\alpha}$. For a very weak dynamo action, i.e. small $D$

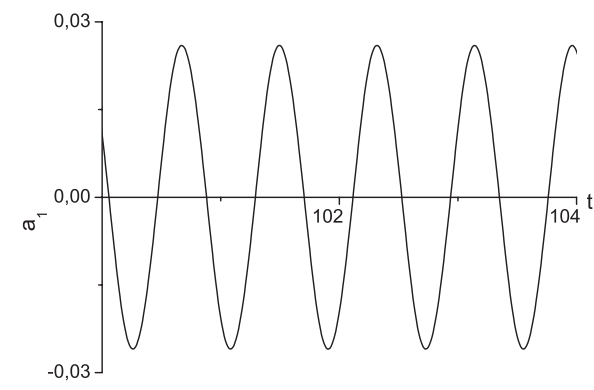

(a)

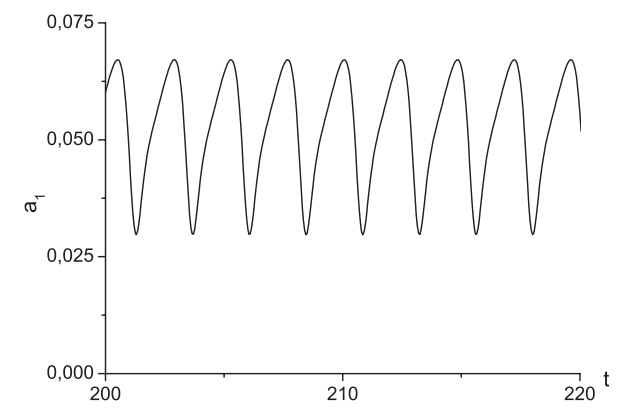

(b)

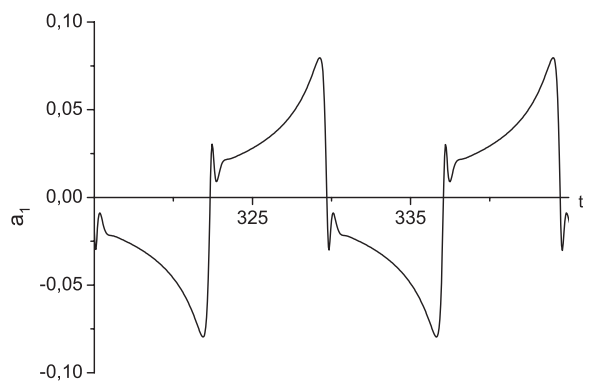

(c)

Figure 2. Various regimes of temporal behaviour for dynamo generated magnetic field (coefficient $a_{1}$ responsible for magnetic moment versus time): a - oscillations, b - vacillations, c dynamo bursts. 
magnetic field decays (corresponding range in synoptic map Fig. 1 is marked by 1). For slightly larger dynamo numbers dynamo dynamo self-excitation occurs, dynamo generated magnetic field first growths manometrically and then becomes steady due to nonlinear dynamo saturation (domain 2 in Fig. 1). Even larger dynamo number results in a nonlinear regime with so-called vacillations, i.e. periodic almost harmonic magnetic field evolution with nonzero mean value (domain 3 in Fig. 1; form of this solution is shown in Fig. 2a. Further enlargement of $D$ results in a periodic however highly nonharmonic temporal evolution known as dynamo bursts (domain 4 in Fig. 1). Dynamo generated magnetic field grows slowly then suddenly and rapidly decay up to zero, changes its sign and then grows slowly again with the opposite sign until next sign reversal restore initial magnetic configuration (Fig. 2c). Such temporal behaviour of dynamo generated magnetic field is known from dynamo experiments, e.g. Berhanu et al. (2007).

If dynamo action becomes even larger it becomes less effective so magnetic field decays again (domain marked by 1a in Fig. 1) however enlarging $D$ further we obtain almost harmonic oscillations with zero mean (domain marked 5 in Fig. 1).

We identify domain 5 in Fig. 1 with the dynamo parameter range responsible for the cyclic solar activity while the parameter range 3 looks similar to the normal behaviour of dipole geomagnetic field (almost steady component and moderate variations). In particular, magnetic moment represented in the model by $a_{1}$ changes its sign each oscillation (domain 5) however keeps its sign in course of vacillations (domain 3).

\section{Grand minima and reversals}

Let us consider now what happens if the averaged value of the fluctuating $\alpha$-coefficient corresponds to a dynamo regime located in the domain 5 and one need an $\alpha$-fluctuation as large as several its standard deviations to move the dynamo regime in the domain $1 \mathrm{a}$ where magnetic field decays. Fluctuations required to shift the dynamo regime in domain 1a being quite large occur rarely so after many normal activity cycles something like a Grand minima occurs. Moss et al. (2008) and Usoskin, Sokoloff \& Moss (2009) investi-

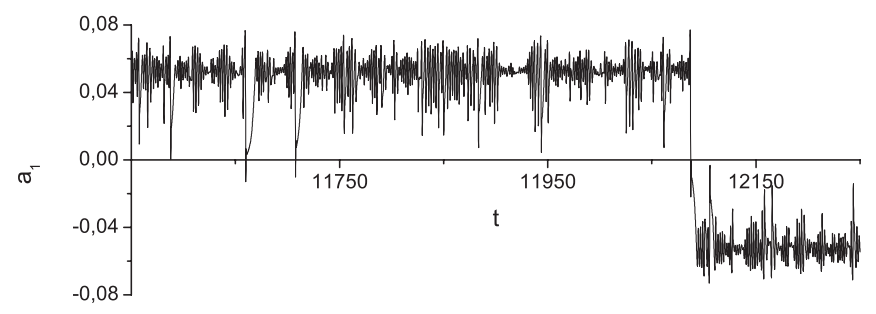

(a)

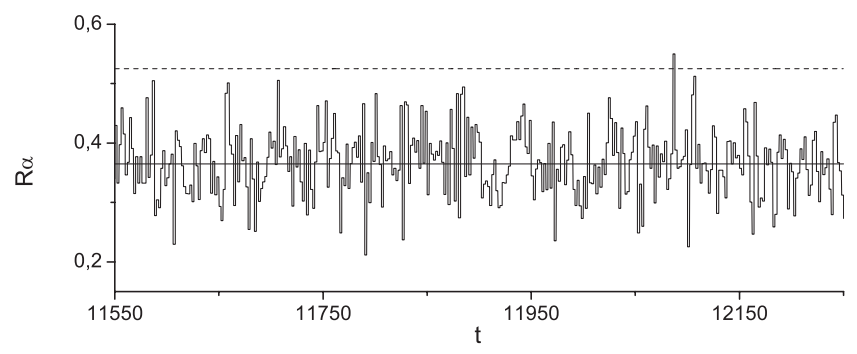

(b)

Figure 3. Scenario for magnetic field reversals: a - evolution of the coefficient $a_{1}$ which represents magnetic moment; b - evolution of $R_{\alpha}$; mean value of $\alpha$ is shown by a horizontal solid line while a horizontal dashed line shows $R_{\alpha}$ which corresponds to transition from domain 3 to the domain 4 in Fig. 1. 
gated (in the framework of Parker migratory dynamo with fluctuating $\alpha$ ) corresponding long-term behaviour of such dynamo to demonstrate that when dynamo governing parameters correspond to the domain 1a magnetic field mimics what happens during the Maunder minimum.

A scenario for magnetic field reversals which resembles the geomagnetic field evolution is slightly more complicated. Let the averaged value of $\alpha$ corresponds to a point in domain 3 , i.e. magnetic field has vacillations. A moderate positive fluctuations of $\alpha$ shifts this point in domain 4 where dynamo bursts occur (Fig. 3b). Further behaviour (Fig. 3a) depends on the scale of this fluctuations and on the phase of the dynamo burst which develops during the fluctuation. If the fluctuation is strong and the burst has enough time during the fluctuation to change sign of dynamo generated magnetic field, then an inversion occurs. If the fluctuation is weaker and/or the dynamo burst occurred changes

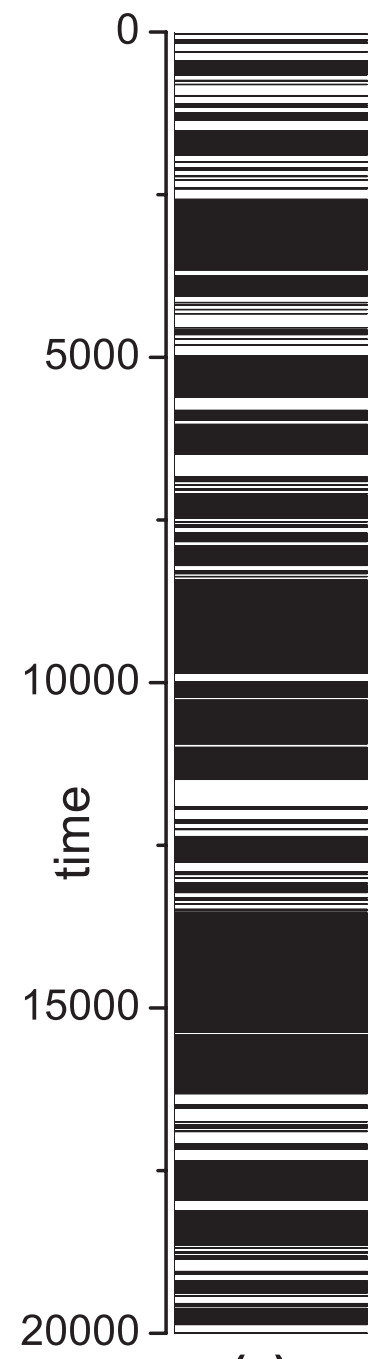

(a)

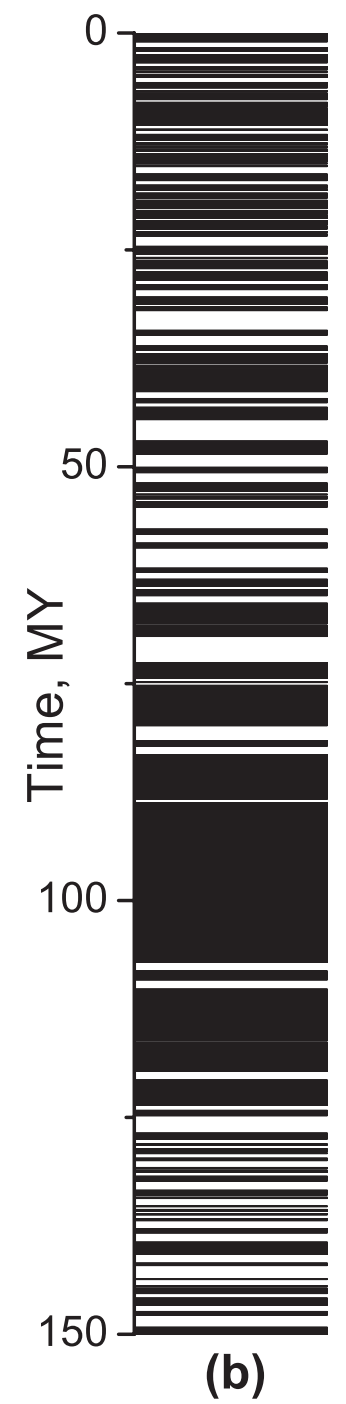

Figure 4. Scenario under discussion gives a geological timescale which is quite similar to that one known from the palaeomagnetic studies: a - simulated geomagnetic timescale; b - geomagnetic timescale for the last 150 million years after Gradstein, Ogg \& Smith (2004). 
sign too late magnetic moment becomes very weak however keeps its sign and its value recovers after the end of the fluctuation. Such episodes are known in the palaeomagnetic studies as excursions. A substantial negative fluctuation of $R_{\alpha}$ can lead to a long epoch without reversals as well as without vacillations because the dynamo regime moves in the domain 2. Such epochs look similar to the long epochs without geomagnetic reversals known in geological history as superchrones.

The temporal evolution of the solution for Eqs. (2.2-2.5) with the averaged values of the dynamo governing parameters located nearby the border, between domains 3 and 4 , demonstrates a sequence of the dynamo generated field reversals. We illustrate this sequence by a diagram known in the palaeomagnetology as geomagnetic time scale, i.e. a column with time as a vertical coordinate where times of one polarity are shown in black while the times of the opposite polarity are shown in white (Fig. 4a). We compare this simulated diagram with a corresponding timescale known from palaeomagnetic studies (Gradstein, Ogg \& Smith 2004) as follows. Note that the dynamical system Eqs. (2.22.5 ) is formulated using a dimensionless time. Of course, palaeomagnetic studies give the instants of the field reversals in dimensional units. We consider, after Gradstein, Ogg \& Smith (2004) the geomagnetic timescale for the last 150 million years where it is most elaborated, choose an interval in the simulated timescale which contains the same number of reversals and presume that the lengthes of both timescales are equal. We present both timescales in Fig. 4. Of course, the timescales can not be identical because at least one of them are taken from a realization of a random process. We note however that the general shapes of both scales are quite similar and conclude that the scenario suggested reproduces basic feature of geomagnetic reversals.

In general, we conclude that the simple dynamical system Eqs. (2.2-2.5) reproduces in specific domains of parametric space basic features of cyclic solar activity as well as evolution of geomagnetic field on geological times including sequence of chaotic field reversals.

\section{Acknowledgements}

DS is grateful for financial support from RFBR, as well as from the organizing committee to participate in the meeting.

\section{References}

Berhanu, M., Monchaux, R., Fauve, S., Mordant, N., Petrelis, F., Chiffaudel, A., Daviaud, F., Dubrulle, B., Marie, L., Ravelet, F., Bourgoin, M., Odier, Ph., Pinton, J.-F., \& Volk, R. 2007, Europhys. Lett., 77, 59001

Brandenburg, A. \& Sokoloff D. 2002, Geophys. Astrophs. Fluid Dyn. 96, 319

Choudhuri, A. R. 2012, this volume

Christensen, U. R., Balogh, A., Breuer, D., \& Glassmeier, K. H. (Eds.), 2010, Planetary Magnetism (Springer)

Frick, P., Stepanov, R., \& Sokoloff, D. 2006, Phys. Rev. E 74, 066310

Gradstein, F., Ogg, J., \& Smith, A. 2004, A Geological Time Scale-2004, Cambridge, Univ. Press.

Hoyng, P. 1993, A\&A 272, 321

Hulot, G., Finlay, C. C., Coustable, C. G., Olsen, N., \& Mandea M. 2010, Space Sci. Rev. 152, 159

Moss, D., Sokoloff, D., Usoskin, I., \& Tutubalin, V. 2008, Solar Physics, 250, 221

Nefedov, S. N. \& Sokoloff, D. D. 2010, Astron. Rep., 54, 247

Olson, P. L., Coe, R. S., Driscoll, P. E., \& Glatzmaier, G. A. 2010, Phys. Earth Planet. Inter., 180,66 
Otmianowska-Mazur, K., Kowal, G., \& Hanasz, M. 2006, A\& A 445, 915

Stepanov, R., Volk, R., Denisov, S., Frick, P., Noskov, V., \& Pinton, J.-F. 2006, Phys. Rev. E 73,046310

Usoskin, I. G., Sokoloff, D., \& Moss, D. 2009, Sol. Phys., 254, 345

\section{Discussion}

Arnab Choudhuri: What kind of diffusivity do you have in your model?

DMitry SokolofF: We assume that turbulent diffusivity is time-independent. In principle it is no problem to include diffusivity fluctuations in the model.

Arnab Choudhuri: How strong fluctuations of the parameters do you need in your model to get a grand minimum?

Dmitry SokolofF: About 10-20\%. 